\title{
Optimization of gadofosveset intravenous injection scheme for coronary MRA: the pharmacokinetics approach
}

\author{
Mark A Ahlman ${ }^{1}$, Fabio S Raman ${ }^{1 *}$, Scott R Penzak ${ }^{5}$, Jianing Pang ${ }^{2,3}$, Debiao Li ${ }^{3}$, David Bluemke ${ }^{1,4}$ \\ From 17th Annual SCMR Scientific Sessions \\ New Orleans, LA, USA. 16-19 January 2014
}

\section{Background}

Towards motion-free diagnostic coronary MRA, lengthy (10-15 min) sequences are required. Conventional GBCAs are unable to sustain steady-state intravascular concentrations long enough to maintain low enough T1 signal for good signal/contrast to noise levels[1]. Arterial and venous mixing of contrast takes time that cannot be used for scanning using our free-breathing self-navigated motion-corrected MRA sequence[2,3]. Gadofosveset has a high intravascular residence time and high relaxivity compared to other GBCAs; therefore, we study various injection parameters to accomplish a low T1 time for the longest period of time, as well as a quick equilibrium time with this agent using a dual bolus/slow infusion injection technique.

\section{Methods}

$\mathrm{X}$ Normal volunteers without a history of cardiac disease and under the age of 40 were selected for scanning on a Siemens Verio 3T scanner following IRB approved informed consent. Gadofosveset was injected with a dual injection protocol using a Spectris Solaris EP (MEDRAD Inc, Pittsburgh, PA, USA) MR injector using a 70\%/\%30 and a $60 \% / 40 \%$ bolus/slow injection technique. Other variables for injection included the the insertion of a 20-30 second pause between bolus and slow infusion, $1.5 \mathrm{~mL} / \mathrm{s}$ vs. $3.0 \mathrm{~mL} / \mathrm{s}$ bolus phase, and single $(0.03$ $\mathrm{mmol} / \mathrm{kg})$ vs double dose $(0.06 \mathrm{mmol} / \mathrm{kg})$. Breath-hold, 4-chamber view, single-slice Modified Look-Locker Inversion Recovery (MOLLI) sequences with 11 heart beats were used to measure absolute $\mathrm{T} 1$ time in the lumen of the left ventricle using QMass MR ver 7.2 (Medis, Raleigh, NC, USA). Unpaired Mann-Whitney t-testing was used to compare variables for statistical significant difference in medians $(\mathrm{p}<0.05)$.

\section{Results}

Shown in Figure 1, the only statistically significant parameter that effects the longest intravascular steady state concentration (within $10 \%$ variation in $\mathrm{T} 1 \mathrm{time}$ ) is the choice of contrast infusion protocol. A $60 \%$ bolus followed by a $40 \%$ slow infusion results in a median infusion time of $>600$ seconds. In Figure 2, we see that a double dose regimen has significant bearing on the speed at which intravascular equilibrium is reached. Whereas, the intertion of a pause, the bolus injection rate, and the proportional volumetric $(70 \% / 30 \%$ vs $60 \% / 40 \%)$ bolus/slow infusion protocol do not.

\section{Conclusions}

Using Gadofosveset as a GBCA for self-navigated motioncorrected MRA, we demonstrated how various contrast injection parameters can be used for optimal steady state intravascular concentration for the longest $\mathrm{T} 1$ relaxivity. Paradoxically, a double dose protocol increases the time to equilibrium, which does not currently have a clear scientific rationale. Multivariate models are pending, which may elicidate these findings. The length of equilibrium is lengthened by using a protocol that has more contrast in the slow

\section{Funding}

This study is funded by the NIH intramural program. 


\section{Variation in Longest Steady State}
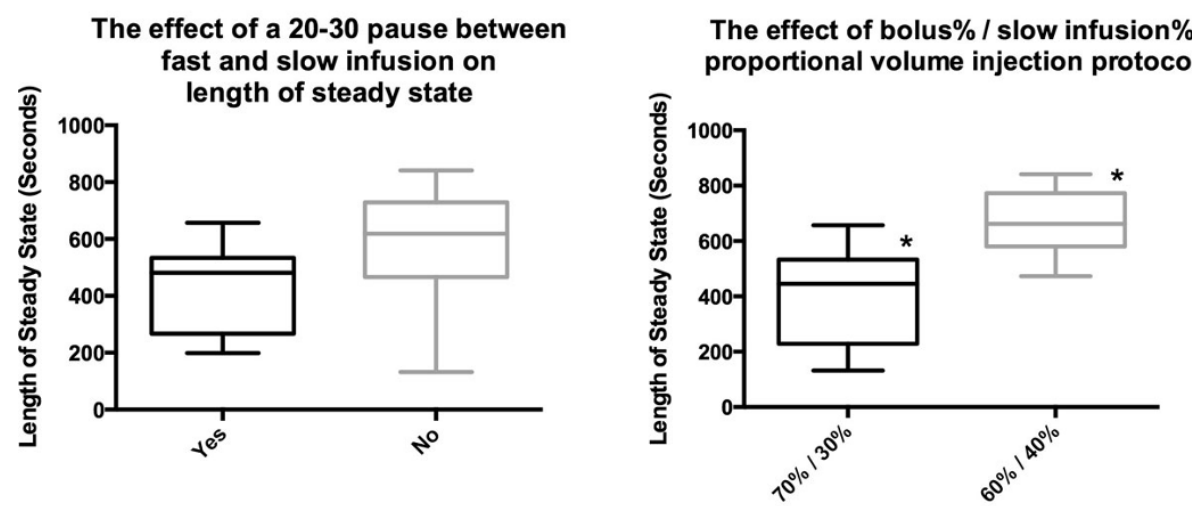

* Mann-Whitney test detected a significant difference between medians $(p=0.0016)$

Figure 1

\section{Variation in time to equilibrium}
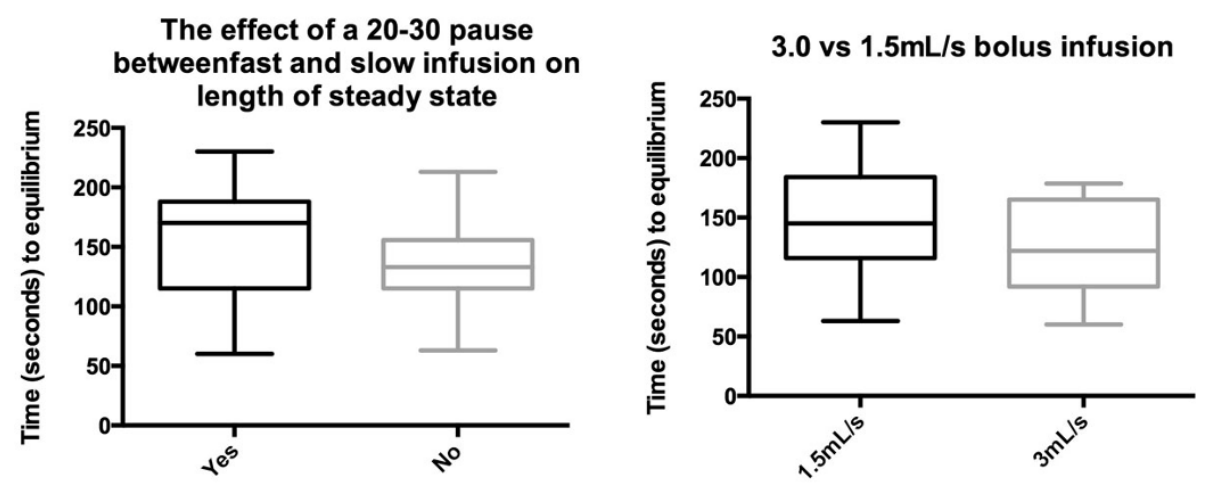

The effect of bolus $\%$ / slow infusion $\%$ proportional volume injection protocol
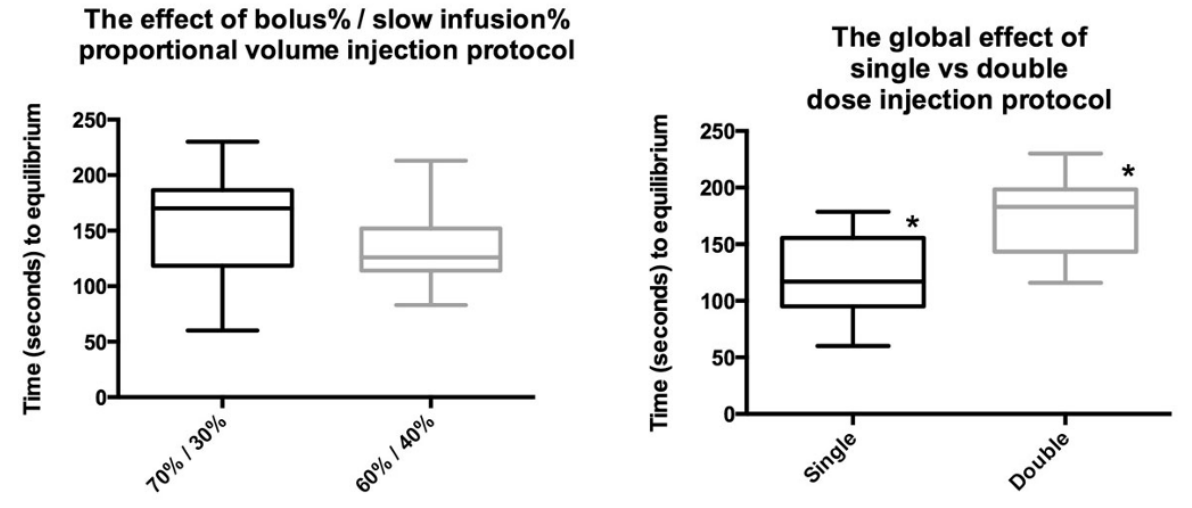

* Mann-Whitney test detected a significant difference between medians $(p<0.001)$

Figure 2 


\section{Authors' details}

${ }^{1}$ Radiology and Imaging Sciences, National Institutes of Health Clinical Center, Bethesda, Maryland, USA. ${ }^{2}$ Departments of Radiology and Biomedical Engineering, Northwestern University, Chicago, Illinois, USA. ${ }^{3}$ Biomedical Imaging Research Institute, Cedars Sinai Medical Center, Los Angeles, California, USA. ${ }^{4}$ Molecular Biomedical Imaging Laboratory, National Institute of Biomedical Imaging and Bioengineering, Bethesda, Maryland, USA.

${ }^{5}$ Clinical Pharmacokinetics Research Laboratory, National Institutes of Health Clinical Center, Bethesda, Maryland, USA.

Published: 16 January 2014

\section{References}

1. Alex Frydrychowicz M, Russe MF, Jelena Bock M, et al: Comparison of Gadofosveset Trisodium and Gadobenate Dimeglumine During TimeResolved Thoracic MR Angiography at 3T. 2010, 17(11):1394-1400.

2. Pang J, Bhat $H$, Sharif B, Fan Z, et al: Self-guided retrospective motion correction (SEGMO) for free-breathing whole-heart coronary MRA with 100\% acquisition efficiency. Journal of Cardiovascular Magnetic Resonance 2012, 14(Suppl 1):M10.

3. Bhat $H$, Ge L, Nielles-Vallespin S, Zuehlsdorff S, Li D: 3D radial sampling and $3 \mathrm{D}$ affine transform-based respiratory motion correction technique for free-breathing whole-heart coronary MRA with 100\% imaging efficiency. Magnetic Resonance in Medicine 2011, 65(5):1269-1277.

doi:10.1186/1532-429X-16-S1-P160

Cite this article as: Ahlman et al:: Optimization of gadofosveset intravenous injection scheme for coronary MRA: the pharmacokinetics approach. Journal of Cardiovascular Magnetic Resonance 2014 16(Suppl 1): P160.

\section{Submit your next manuscript to BioMed Central} and take full advantage of:

- Convenient online submission

- Thorough peer review

- No space constraints or color figure charges

- Immediate publication on acceptance

- Inclusion in PubMed, CAS, Scopus and Google Scholar

- Research which is freely available for redistribution

Submit your manuscript at www.biomedcentral.com/submit
C Biomed Central 